\title{
QRA MODEL BASED RISK IMPACT ANALYSIS OF TRAFFIC FLOW IN URBAN ROAD TUNNELS
}




\begin{abstract}
Road tunnels are vital infrastructures providing underground vehicular passageways for commuters and motorists. Various quantitative risk assessment (QRA) models have recently been developed and employed to evaluate the safety levels of road tunnels in terms of societal risk (as measured by the F/N curve). For a particular road tunnel, traffic volume and proportion of heavy goods vehicles (HGVs) are two adjustable parameters that may significantly affect the societal risk, and are thus very useful in implementing risk reduction solutions. To evaluate the impact the two contributing factors have on the risk, this paper first presents an approach that employs a QRA model to generate societal risk for a series of possible combinations of the two factors. Some combinations may result in F/N curves which do not fulfill a predetermined safety target. This paper thus proposes an "excess risk index" in order to quantify the road tunnel risk magnitudes which do not pass the safety target. The two-factor impact analysis can be illustrated by a contour chart based on the excess risk. Finally, the methodology has been applied to Singapore's KPE road tunnel and the results show that in terms of meeting the test safety target for societal risk, the traffic capacity of the tunnel should be no more than 1,200 vehs/hour·lane, with a maximum proportion of $18 \%$ HGVs.
\end{abstract}

Key words: Impact analysis, quantitative risk assessment model, societal risk, road tunnel, traffic volume 


\section{INTRODUCTION}

Road tunnels are vital infrastructures providing underground vehicular passageways for commuters and motorists. They contribute to transportation systems, both economically and practically, because they enhance capacity and accessibility. However, fatal accidents occurring in road tunnels may result in catastrophic consequences. In order to evaluate the risks associated with these complex hazardous installations, the quantitative risk assessment (QRA) methodology, consisting of event trees, fault trees and consequence estimation models, was proposed in the 1970s and has been applied extensively since then. ${ }^{(1-3)}$ This is because it systematically deals with all possible hazards, the magnitudes (severities) of possible adverse consequences and the likelihood (frequency) of each possible scenario. Based on this methodology, several different QRA models have been developed by different countries to assess the risks of their road tunnels. ${ }^{(1)}$ For some newly constructed urban road tunnels, configurations, geometries, electrical and mechanical (E\&M) systems (e.g. tunnel ventilation systems), traffic volumes, accident frequencies, and other factors, may vary from one section to another. These types of tunnels, characterized by non-uniform tunnel parameters, are referred to as non-homogeneous urban road tunnels by Meng et al. (2010). ${ }^{(4)}$ A new QRA model named QRAFT was recently developed for non-homogeneous urban road tunnels and has been applied successfully by Singapore's Land Transport Authorities (LTA) to road tunnels there. ${ }^{(4-7)}$

Various risk indices are applied to evaluate the risks of hazardous installations in risk analysis models. ${ }^{(8-10)}$ According to The Handbook of Tunnel Fire Safety ${ }^{(11)}$, the consequences of tunnel incidents may include: (1) fatality, (2) injury, (3) property loss, and (4) disruption of operations, with the number of fatalities being the predominant concern of the LTA. The comprehensive literature review also shows that the majority of the existing QRA studies on road tunnels only take the number of fatalities into account. ${ }^{(1,2)}$ In addition, all the preventive and/or protective systems (e.g. fire detection systems, tunnel ventilation systems) installed in a road tunnel aims specifically to reduce number of fatalities. In fact, the injury and property loss are the concerns of insurance company from which the victims claim money, not the concerns of tunnel authorities. Therefore, this study also uses the societal risk (represented by Frequency/Number of fatalities 
curve), from their perspective of land transport authorities, to measure the consequence without taking into consideration the other concerns. Societal risk is a widely used and well recognized index, reflecting the relationship between the frequency and number of fatalities on a double logarithmic scale. ${ }^{(12)}$ Most countries have chosen an upper bound for societal risk as a safety target for their road tunnels. ${ }^{(2,13)}$ If the F/N curve generated by a QRA model is below the chosen safety target, the road tunnel is considered safe. Otherwise, risk reduction measures need to be implemented.

Under the QRA model, the risk of a given road tunnel is determined by its geometries, traffic volume, vehicle composition, hazmat transportation, E\&M systems, the distance between evacuation exits, and other parameters. The tunnel geometries and safety provisions are designed at the planning stage. Once the tunnel is open to traffic, these parameters are considered to be un-adjustable - it would be difficult, if not impossible, to adjust these parameters to reduce the risks.

By contrast, critical components of traffic flow can be controlled conveniently through the use of entry controls and traffic regulations. Total traffic flow has an important bearing on societal risk because as the traffic volume in a road tunnel increases there tends to be an increase both in the frequency of accidents and in the number of injuries and fatalities in any given accident ${ }^{(14,15)}$. The number of Heavy Goods Vehicles (HGVs) in proportion to total traffic is an important factor within this general pattern, both because HGVs as such increase the risk of accident, and because an accident involving an HGV tends to be more severe in terms of fatalities than one involving only smaller vehicles. These elements are recognized in Singapore, where prior notice must be given to the Transport Authority (LTA) before an HGV can enter a road tunnel, and overall traffic flow through the tunnel can be controlled using the normal signaling system.

The above discussion implies that traffic volume and proportion of HGVs are two critical contributing factors to the societal risk of road tunnels. It is thus important to capture a picture of how these two factors affect societal risk. Towards this end, a risk impact analysis approach is proposed, not only to support the design considerations of new tunnels by varying these two factors (planning data), but also to evaluate various road tunnel traffic control schemes and HGV transport regulations, which may provide 
helpful information to decision makers. In addition, given a combination of the traffic volume and the proportion of HGVs, the F/N curve generated by a QRA model may not fulfill a predetermined road tunnel safety target. In this case, we need an index to measure how far the risk is from the safety target. Accordingly, the "excess risk index" is defined to quantify the danger levels of road tunnels relative to the safety target. Based on this index, a contour chart is plotted, incorporating possible combinations of the two contributing factors. This chart could further facilitate LTAs' decision making.

The contributions of this paper can be summarized as follows. Firstly, an impact analysis methodology for road tunnels, based on QRA models, is proposed to quantify the impact of the traffic volume and the proportion of HGVs on the risk, which will support tunnel designers' decision making, as well as the determination of traffic control strategies. Secondly, the excess risk index allows us to evaluate the relative danger levels of tunnels which do not meet the safety target. The contour chart based on this may further facilitate risk reduction plans. Thirdly, applications of this approach to the Singapore KPE road tunnel demonstrate the usefulness and effectiveness of the proposed methodology using actual data.

The remainder of the paper is organized as follows. In Section 2, we briefly introduce the QRAFT model and the definition of societal risk. This is followed by the definition of the excess risk index and excess risk-based impact analysis procedure in Section 3. Section 4 presents the application of the methodology to the KPE road tunnel in Singapore. The final section contains conclusions and discussions.

\section{QRAFT MODEL AND SOFTWARE}

\subsection{QRAFT Model}

Singapore's road tunnels cannot be homogeneously examined without taking the multifarious geometric layouts of its tunnel sections into account and the existing QRA models thus cannot be applied to Singapore road tunnels. Therefore, the National University of Singapore (NUS) and the LTA of Singapore jointly developed a QRA model for the country's road tunnels (non-homogeneous road tunnels) in 2009, called the

QRAFT model. ${ }^{(4,5)}$ The procedure of the model is summarized as follows. First, a tunnel segmentation principle is proposed to divide the non-homogeneous road tunnels into a 
number of homogeneous road tunnel sections. Second, aiming at each individual homogeneous tunnel section, a QRA model is built to estimate the risk indices for the tunnel section. Third, risk integration principles are applied to estimate the risk indices for the overall road tunnel by combining risks of various tunnel sections based on their weights (travel rate in terms of vehicle kilometer per year). The model consists of seven top events (fire, flood, toxic gases generated by traffic congestion, tunnel collapse, chain collision, explosion, and spillage due to hazmat), seven event trees, seven fault trees and nine consequence estimation methods. Nineteen E\&M systems are taken into account in the proposed QRA model. The detailed model is given in Meng et al. (2010). ${ }^{(4)}$. The work was conferred with the Singapore Ministry of Transportation Minister's Innovation Award 2009.

The key input parameters required for the QRAFT model can be categorized into four types: traffic flow parameters, tunnel user characteristics, tunnel geometries, and parameters associated with the tunnel's E\&M systems. Traffic parameters include traffic volume, accident frequencies, vehicle composition (the different proportions of various types of vehicles), the headway distances and the fraction of hours that are peak/off-peak, among others. Tunnel user characteristics refer to variables such as the reaction times of tunnel users, the speeds of tunnel users, the proportions of tunnel users of various ages, and the proportion that are experienced drivers. Statistics on Singapore residents and/or drivers are used to deduce these values. Tunnel geometries relate, among other things, to the distance between two consecutive emergency exits, the number of lanes, tunnel sectional area, and tunnel height. The parameters associated with the tunnel's E\&M systems are the functional parameters of those systems, the reliability parameters, and the probabilities of those systems failing to work.

\subsection{Societal Risk and Safety Targets}

Societal risk is defined as the frequency of incidents with more than a given number of fatalities due to the realization of specified hazards. ${ }^{(12)}$ It can be represented graphically in the form of an $\mathrm{F} / \mathrm{N}$ curve. This measure is generally accepted in the quantitative risk assessment of road tunnels. ${ }^{(1,2)}$ Let $F(N)$ denote the estimated annual frequency of incidents incurring $N$ or more fatalities. We thus have: 


$$
F(N)=\sum_{i=N}^{M} F_{i}
$$

where $F_{i}$ is the estimated annual frequency of scenarios with $i$ fatalities; $M$ is the upper limit of the number of fatalities per incident.

The following upper bound curve for $F(N)$ has been adopted by various countries as the safety target: ${ }^{(13,16)}$

$$
F(N) \leq \frac{C}{N^{k}}
$$

where parameters $k$ and $C$ specify the steepness and intercept of the safety target. Alternatively, Equation (2) can be represented by:

$$
k \log (N)+\log (F(N)) \leq \log (C)
$$

It should be noted that $k$ represents a slope, i.e. the gradient of the safety target, and $C$ denotes an intercept, i.e. the constant value that determines the position of the target. Different combinations of $k$ and $C$ express various degrees of strictness. As a result, different countries may propose different targets. For example, the $k$ and $C$ values adopted by the Netherlands for chemical installations are $C=10^{-3}$ and $k=2$. $^{(13)}$

Societal risk is a convenient measure for decision makers to judge whether a road tunnel meets a predetermined safety target. However, it only provides a binary judgment and does not reflect the degree of danger quantitatively. Horn et al. (2008) proposed another measure, defined as the total extent to which the constraint is violated ${ }^{(10)}$. In the notation of the present paper, this is defined as:

$$
v=\sum_{N=1}^{M} \max \left\{F(N)-\frac{C}{N^{k}}, 0\right\}
$$

\subsection{QRA Software Tool}

A user-friendly QRA software tool was developed using the Object-Oriented Design (OOD) method to implement the QRAFT model. The design and implementation of the software are illustrated by Meng and Qu (2010). ${ }^{(7)}$ It is coded in C\#, and uses a Microsoft Access database and XML files to manage the data. Its user-friendly interface and the database technique applied make the job of saving and editing input parameters effortless. It thus enables us to perform a sensitivity analysis based on the proposed model. The 
QRAFT QRA model and software tool have already been used by Singapore's LTA to evaluate the road tunnel risk for the Marina Coastal Expressway (MCE). ${ }^{(6)}$

\section{RISK IMPACT ANALYSIS METHOD}

In this section, we first propose an excess risk index in order to quantify the magnitude of risk for road tunnels which do not meet safety targets. Based on the proposed index, an excess risk-based risk impact analysis is presented, to examine how the traffic flow parameters influence risks.

\subsection{Excess Risk Index}

Equation (4) is a good index to measure the degree of danger quantitatively. In mathematical terms, the weighting for severity appears to be linear in Horn's index (cumulative quantities). However, land transport authorities pay much more attention to high-consequence events. Therefore, the high-loss events, even if their frequencies of occurrence are very low, should be given higher weight. Accordingly, following Horn et al.'s work, we propose a risk index, the "excess risk index", defined as the weighted summation of the distances between the predetermined safety target and the $\mathrm{F} / \mathrm{N}$ points which are above the safety target.

In reality, the concept of excess risk index is also similar to the well-known risk index named Conditional Value at Risk (also called mean excess risk) in finance studies ${ }^{(17,18)}$. The relationship between societal risk and excess risk is just the same as that between Value at Risk and Conditional Value at Risk. In both cases, an exceedance measure reports the extent to which a predetermined safety target is violated. In these measures, a quadratic weighting is given to high-consequence events, compared to aggregates of smaller events. As we mentioned earlier, land transport authorities also pay much more attention to high-consequence events compared to low-consequence ones. In the present application, it is therefore appropriate to use a quadratic risk index rather than a form such as Equation (4).

Figure 1 depicts an F/N curve generated by the QRAFT software tool. The diamonds are generated in the case where traffic volume is 1,800 vehs/hour-lane. The asterisks are generated in the case of 1,600 vehs/hour-lane. As per the safety target, shown by the 
straight line, both scenarios are unacceptable since both curves have some points above the straight line. However, the F/N curve with the diamonds represents a much more dangerous scenario than does the asterisks curve (all the diamonds are higher than the asterisks (e.g. $F_{1800}(3)=1.5 \times 10^{-4}>F_{1600}(3)=8.8 \times 10^{-5}$ ).

The mathematical expression of the excess risk index is shown by Equation (5).

$$
S_{e}=\sum_{N=1}^{M} \max \left\{\left(F(N)-\frac{C}{N^{k}}\right) \times N, 0\right\}
$$

For an acceptable F/N curve, $S_{e}$ takes the value of 0 . As shown in Figure 1, with increasing numbers of fatalities, the F/N curve shifts to the right, while with increasing frequencies it shifts upwards, and wherever $F(N)$ surpasses the target curve, the excess risk value becomes non-zero. In reality, excess risk basically refers to the area of any regions where $F(N)$ lies above the line of the predetermined safety target.

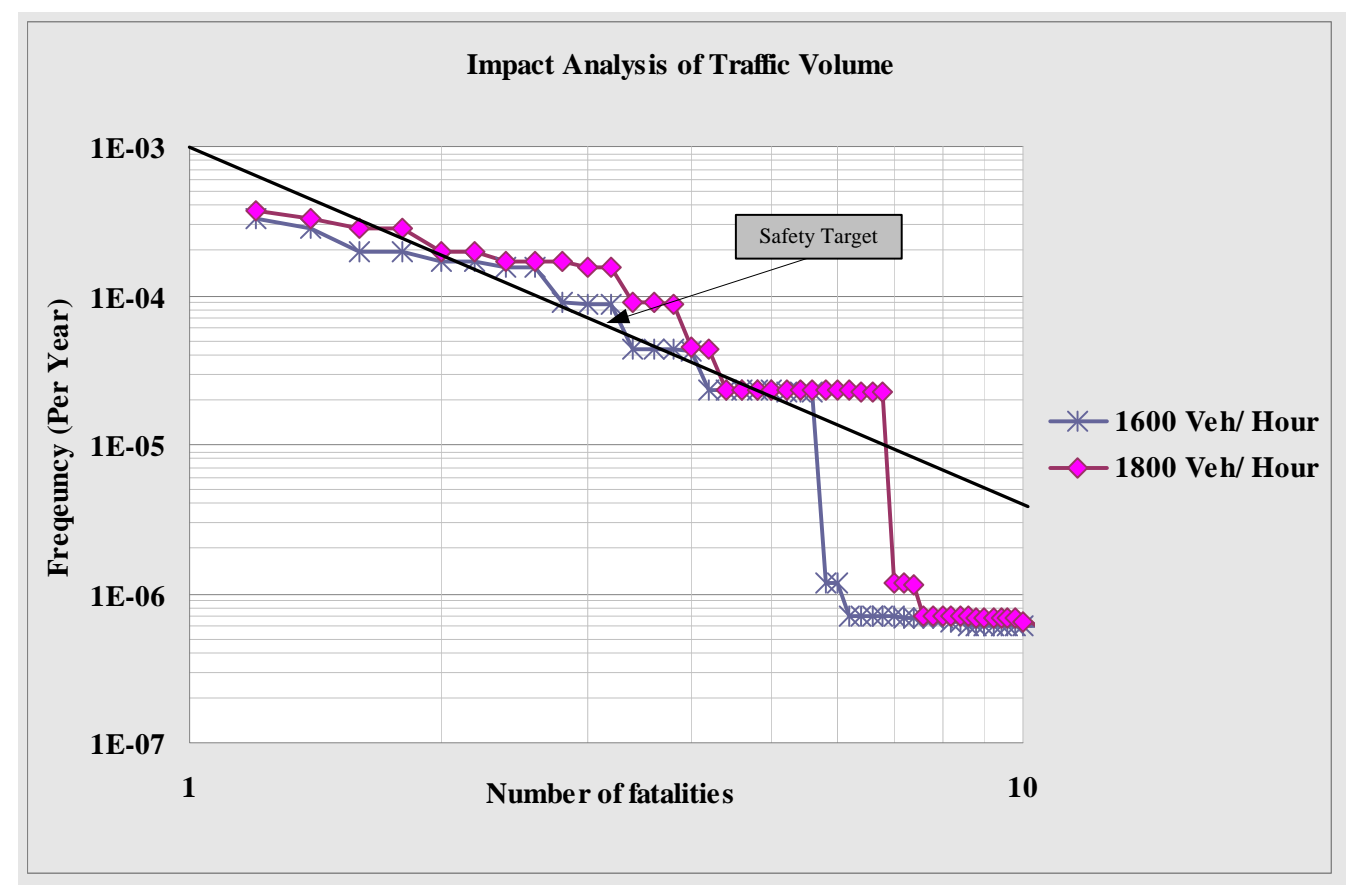

Fig. 1: An F/N curve example

\subsection{Excess Risk Index-Based Risk Impact Analysis}

A risk impact analysis of the two contributing factors, traffic volume and proportion of HGVs, to the societal risk of a road tunnel can be implemented easily using the 
QRAFT software tool. The F/N curve can be generated using the tool on a case by case basis. The risk impact analysis procedure works as follows. Firstly, determine the ranges of traffic volume $\left(X_{i}\right)$ and proportion of $\operatorname{HGVs}\left(Y_{j}\right)$ and discretize these ranges. The range of traffic volume may be assumed to be between 1,000 and 1,800 vehs/hour-lane, based on historical data, and the step is taken to be 200 vehs/hour-lane, based on expert judgment. The examined traffic volumes are thus taken to be 1,000, 1,200, 1,400, 1,600, and 1,800 vehs/hour-lane. Further details are given in Section 4. The values of the other input parameters required in the QRA model (as described in Section 2.1) can be acquired from historical data and the design documents of the road tunnels. These values are held constant in this risk impact analysis.

Next, a quantitative risk analysis is performed for all possible combinations of the two major contributing factors (traffic volume and proportion of HGVs). The societal risk for all combinations is generated using the QRA software tool and the excess risk $\left(R_{e}\right)$ can thus be calculated using Equation (5). Excess risk can thus be considered as the output of the various combinations of the two contributing factors.

Finally, an excess risk contour chart is introduced to illustrate the changing pattern of excess risk. We plot points (combinations of the two contributing factors) which produce the same excess risk, that is, for a given value of excess risk $\alpha$, we plot the points $\left(X_{i}, Y_{j}\right)$ where $R_{e}\left(X_{i}, Y_{j}\right)=\alpha$. Then, the B-spline curve fitting method, a process of constructing a smooth curve with the best fit to a series of data points, is adopted to generate a smooth contour line. Finally, the excess risk contour chart is drawn using these curve fitting methods for varying values of $\alpha$. Figure 2 illustrates the two-factor impact analysis procedure. 


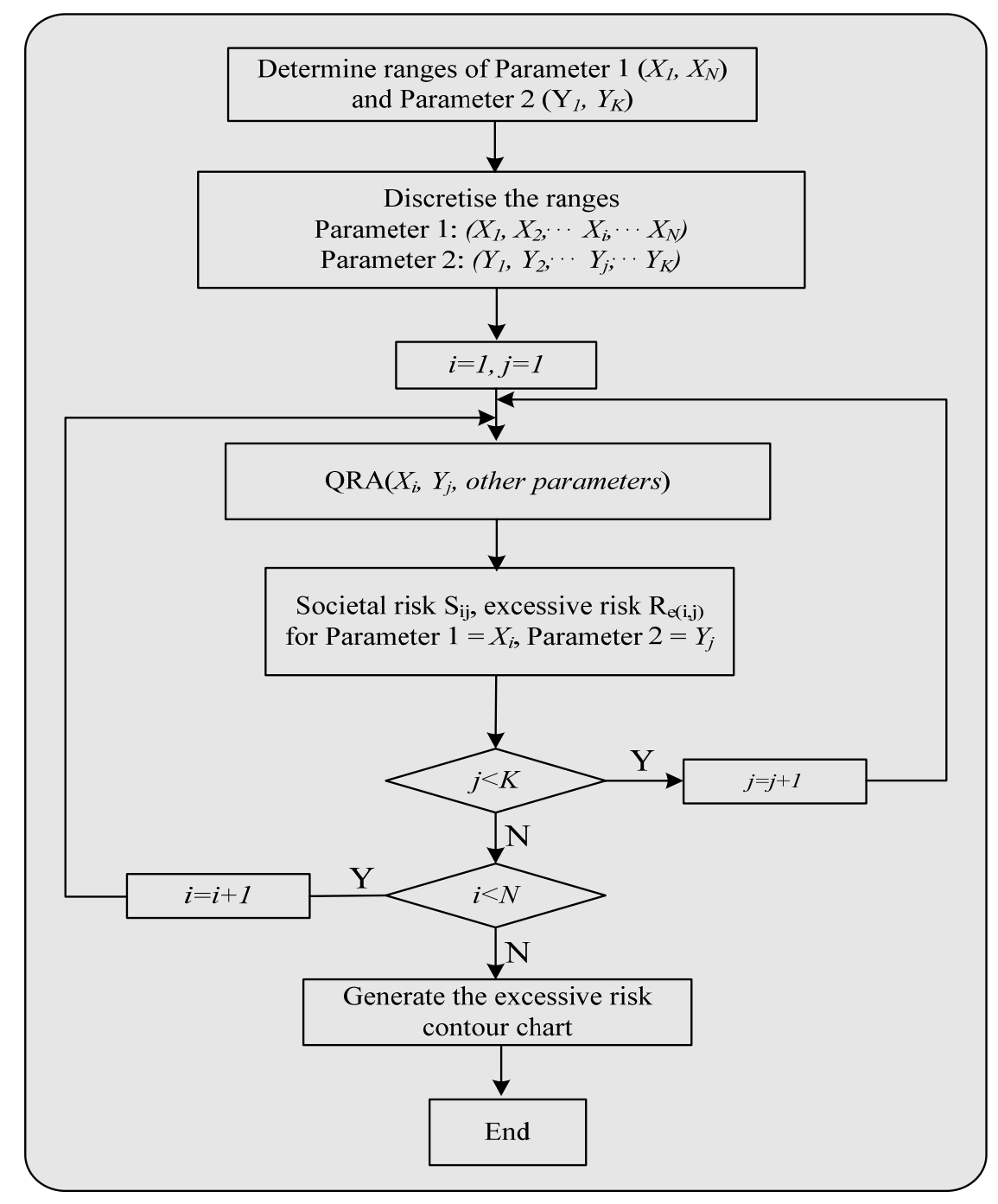

Fig. 2: Two-factor sensitivity analysis procedure

It should be pointed out that this method and the proposed index could also be applied to analyze the impact of other input parameters, and could also be generalized to analyze three or more parameters as well.

\section{RISK IMPACT ANALYSIS FOR SINGAPORE ROAD TUNNELS}

We have applied this methodology in conjunction with LTA of Singapore to evaluate the effect of the two above-mentioned important contributing factors: traffic volume and the proportion of HGVs. Thereby, the traffic capacity and acceptable proportion of HGVs in terms of risk in the three existing non-homogeneous urban road tunnels in Singapore have been derived. 
The Kallang/Paya Lebar Expressway (KPE) in Singapore, shown in Figure 3, has a total length of twelve kilometers, and is 36 meters wide. Nine kilometers of the expressway is built underground as a road tunnel, serving the growing traffic demands of the north-eastern sector of Singapore. It is the longest road tunnel in South East Asia. The KPE road tunnel is a dual three-lane underground passageway and has nine entry slip roads, eight exit slip roads and six longitudinal ventilation buildings. The cross-sectional area of the tunnel is around 306 meters squared. Its accident frequency is 560 per year on average, according to the historical data (2001-2006). The distance between emergency exits is one hundred meters. The critical ventilation velocity, that is the minimum speed of the longitudinal ventilation, is six meters per second. There is a twenty-four hour manned operation control centre (OCC) in one of the ventilation buildings and an unmanned hot standby OCC located in another ventilation building. The major E\&M systems of the KPE tunnel include a tunnel ventilation and environmental control system, a fire protection system, an electrical system, an integrated traffic and plant management system and a communications system.

The QRAFT model and software tool are utilized to conduct the impact analysis. It should be pointed out that the societal risk for the overall road tunnel is aggregated across the individual sections of the tunnel system using the QRAFT software and the risk integration principle is illustrated in Meng et al. ${ }^{(4)}$ Note that Horn et al. also proposed approaches to combine the risks of various sections. ${ }^{(10)}$ 


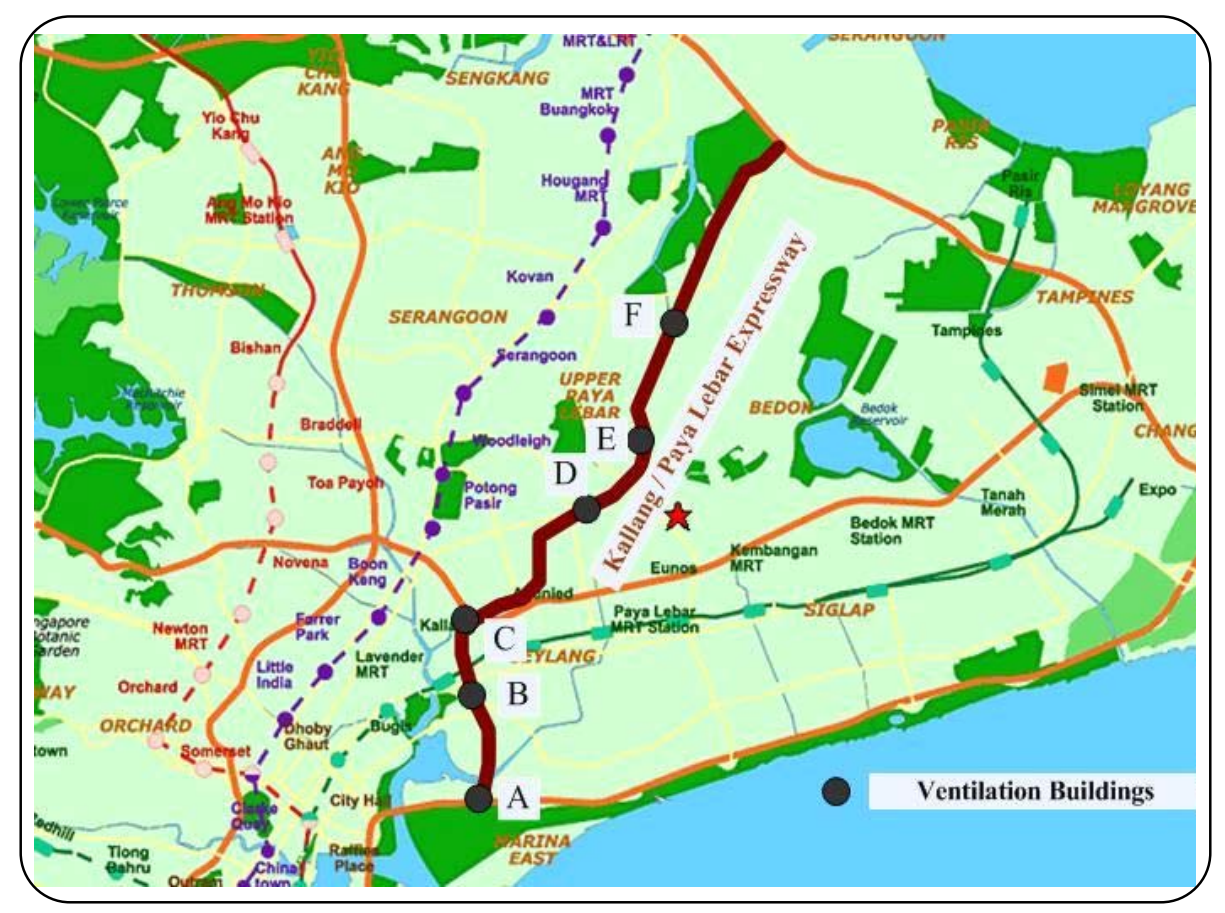

Fig. 3: KPE road tunnels in Singapore

\subsection{Traffic volume impact analysis}

In order to quantify the impact on societal risk of the road tunnel's traffic volume, measured as total number of vehicles at peak hour, an impact analysis is performed. All the other input parameters (default values) are based on operational data collected from Singapore's KPE road tunnel. As mentioned earlier, the traffic volume varies from 1,000 to 1,800 vehs/hour-lane. The $\mathrm{F} / \mathrm{N}$ curves associated with different traffic volumes are shown in Figure 4. A safety target of $10^{-3} / \mathrm{N}^{2}$ is adopted in this case study.

Figure 4(a) depicts the F/N curves for traffic volumes varying from 1,000 to 1,400 vehs/hour-lane. In these three scenarios, the KPE tunnel can be considered safe based on the chosen safety target. Figure 4(b) shows the F/N curves for traffic volumes of 1,600 and 1,800 vehs/hour-lane. It can be seen that these two scenarios are not acceptable based on the selected safety target. Therefore, it can be concluded that the maximum tolerable traffic volume of the KPE road tunnel is 1,400 vehs/hour·lane. 


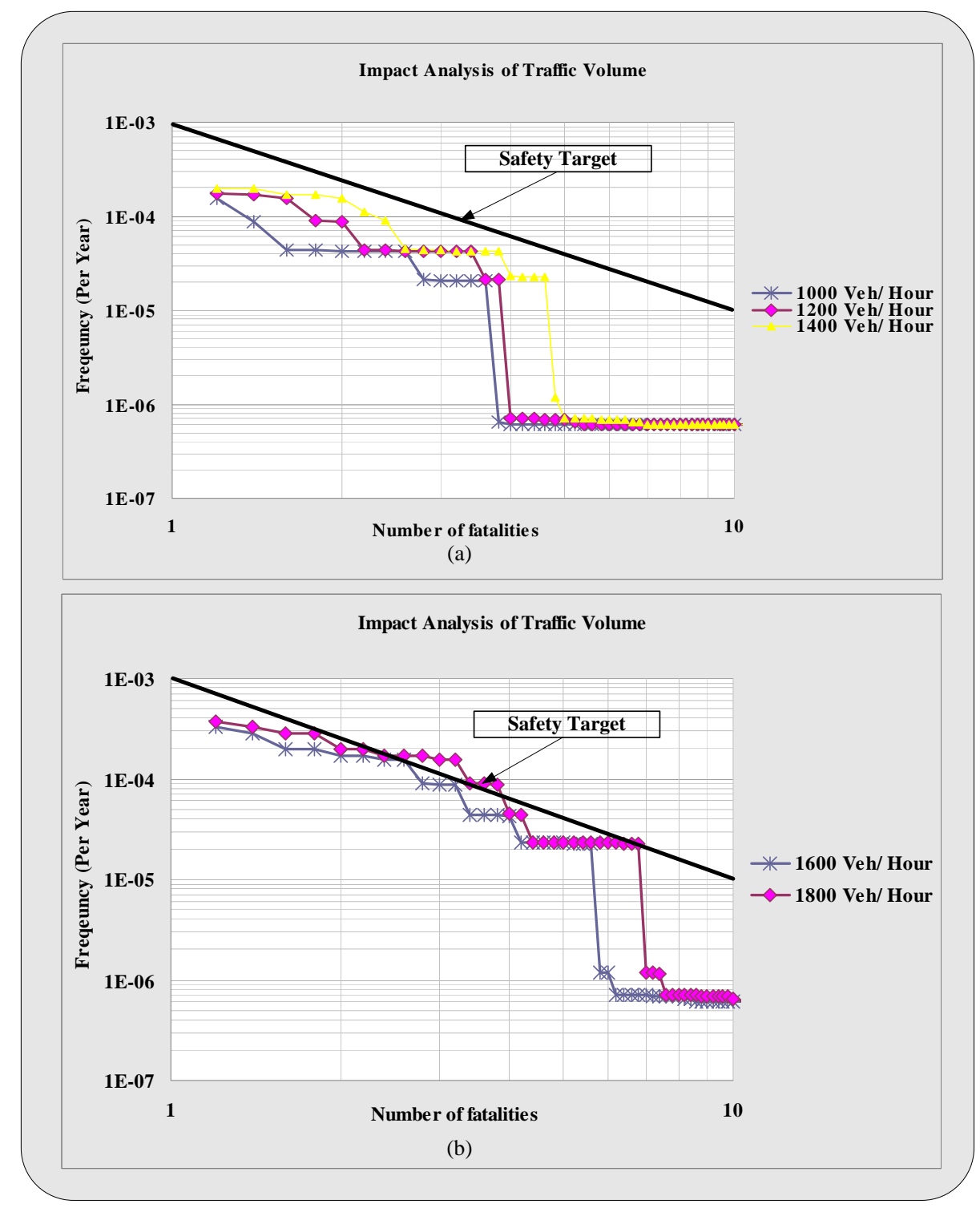

Fig. 4: Two F/N curve diagrams for the KPE road tunnel

\subsection{Impact Analyses on the Proportion of HGVs}

As mentioned in the Introduction section, according to the Road Traffic Act of Singapore, a form of notice is required to be submitted to the LTA of Singapore before an HGV enters the KPE road tunnel. This impact analysis will determine the maximum proportion of HGVs that is safe, supporting the LTA's decisions regarding how many HGVs it allows to enter the tunnel. The proportion of HGVs ranges from 5 to $30 \%$. Meanwhile, the traffic volume takes values of between 1,200 and 1,800 vehs/hour·lane. 
Figures 5(a) to 5(d) represent the F/ $\mathrm{N}$ curves for scenarios with traffic volumes of 1,200, 1,400, 1,600, and 1,800 vehs/hour-lane, respectively, for varying proportions of HGVs. Figure 5(a) shows that if the traffic volume is relatively low, even with a $30 \%$ proportion of HGVs, the F/N curve stays below the safety target. However, at 1,400 vehs/hour-lane (which is likely, due to the densely populated nature of Singapore), even 15\% HGVs could impose a significant threat to the tunnel's users, since some of the $\mathrm{F} / \mathrm{N}$ points exceed the safety target (Fig. 5(b)). 


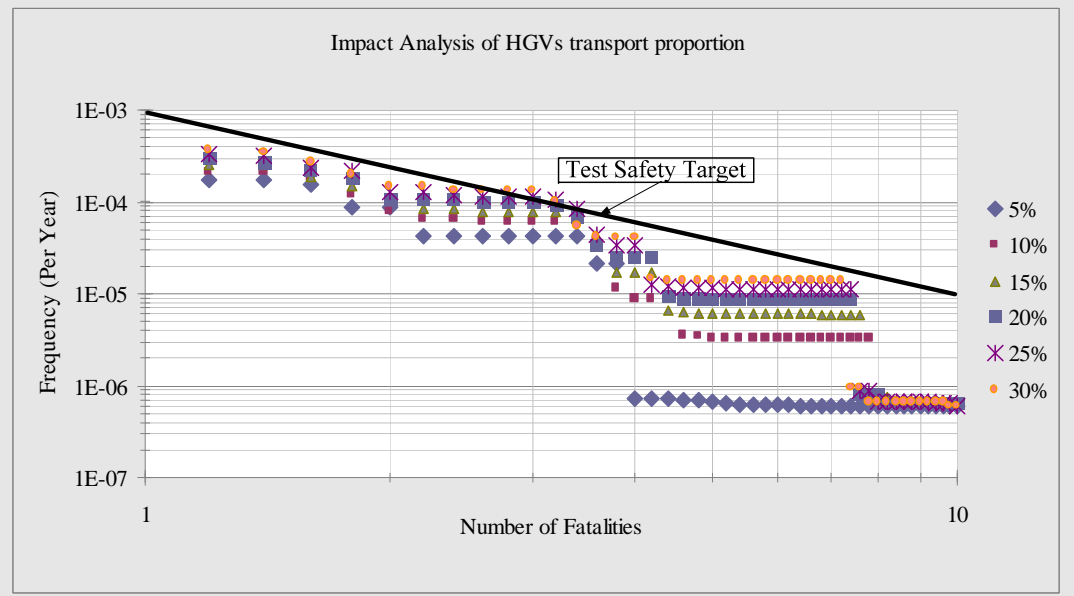

(a) Traffic Volume $=1200$ vehs per hour per lane

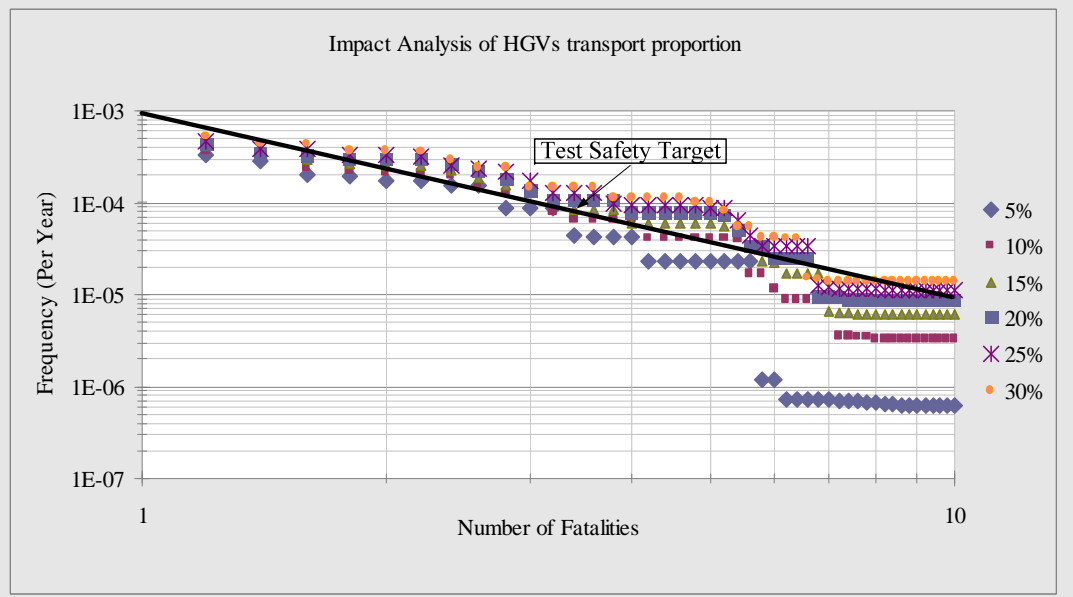

(c) Traffic Volume $=1600$ vehs per hour per lane

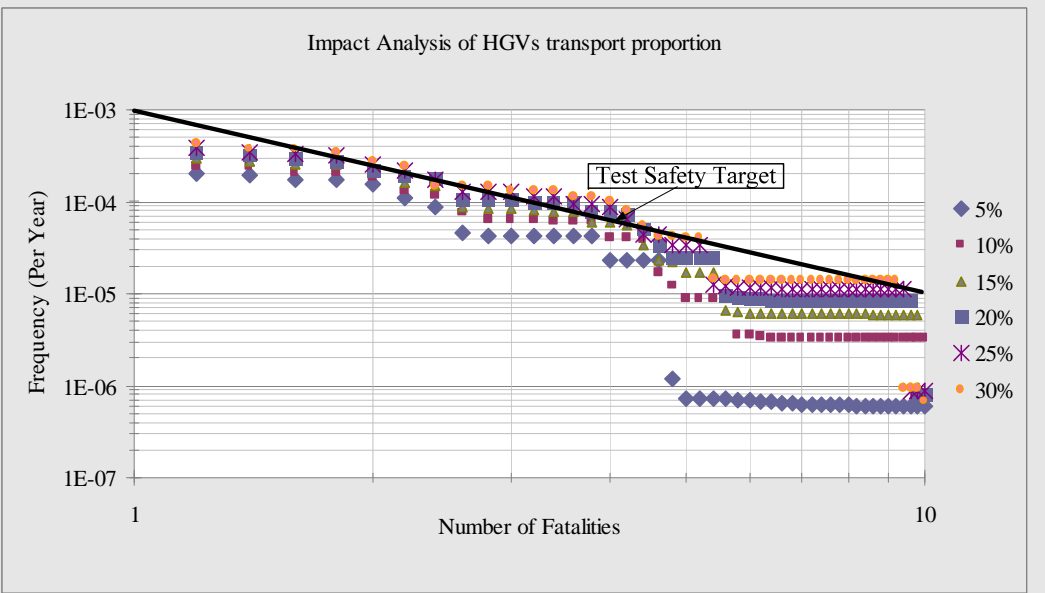

(b) Traffic Volume $=1400$ vehs per hour per lane

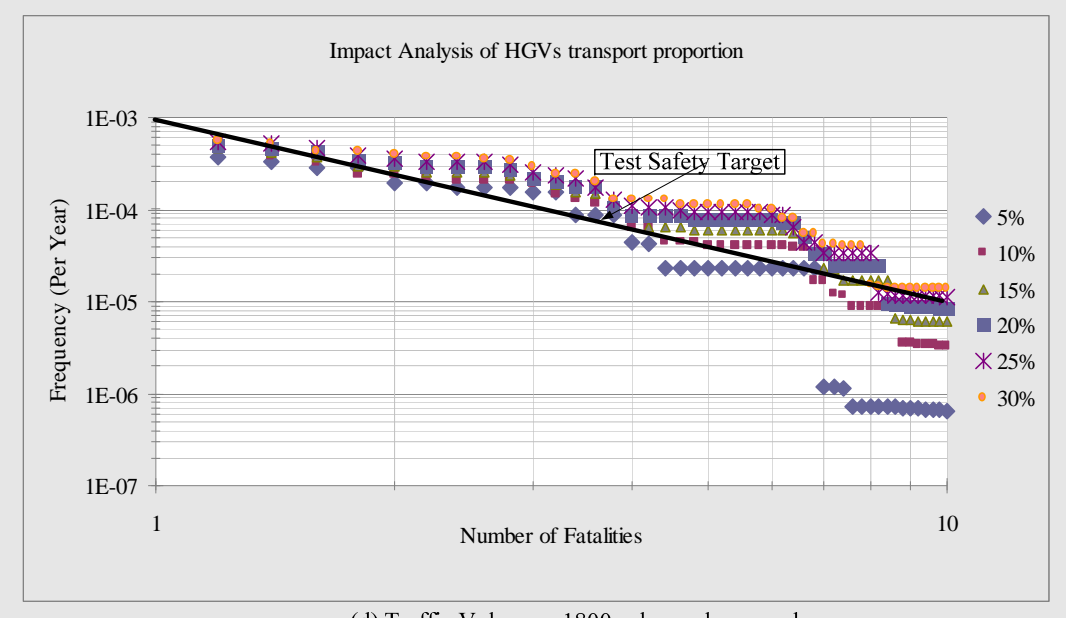

(d) Traffic Volume $=1800$ vehs per hour per lane

Fig. 5: Four F/N curve diagrams of the KPE road tunnel 


\subsection{Excess Risk Index Contour Chart}

The above impact analysis indicates that traffic volume and the proportion of HGVs have a significant impact on the tunnel risk. Therefore, both parameters should be taken into consideration in the impact analysis. For each combination of these two parameters, the excess risk index is calculated using the QRAFT software tool. The excess risk index-based contour chart is drawn by varying the traffic volume from 1,000 to 1,800 vehs/hour/lane in steps of 200 and the proportion of HGVs from $5 \%$ to $30 \%$ in steps of $5 \%$. After obtaining the excess risk value for all thirty possible combinations of the two variables, the risk contour chart is drawn using the curve fitting method (as described earlier). The contour chart is shown in Figure 6. Region 1 is considered to be the safe region. The excess risk index will become bigger as the traffic volume and proportion of HGVs increase. This contour chart can assist decision-makers in deciding on the most appropriate combination of traffic volume and proportion of HGVs for any given safety target.

As the population of Singapore increases, more road tunnels will be built due to the need for more efficient land use. Furthermore, more HGVs pass along Singapore's expressways than along other countries' roads, due to the need to transport containers to and from the port of Singapore, the busiest container port in the world. It is thus important to determine the most suitable combinations of the two most important contributing factors to societal risk. Considering the urban nature of Singaporean road tunnels, traffic volume tends to be at the higher end of the range that we have considered in our impact analysis. This means that the road tunnels may be operating near to the risk contour line 0 , which shows the maximum allowable traffic volume based on the selected safety target. If the traffic volume or the proportion of HGVs increases, then the risk index may increase beyond the risk contour line 0 , which will no longer satisfy the safety target. If effective operational procedures are implemented, this would help to reduce the transportation of HGVs through road tunnels, then this would effectively shift the excess risk index from risk contour line $10^{-5}$ to risk contour line 0 and thus the chosen safety target would still be satisfied. For example, if the tunnel were operating with 1,200 vehs/hour/lane and 20\% HGVs (Point A in Figure 6), the operational status would be unsafe and risk reduction solutions would need to be implemented. Based on Figure 6, the LTA could either reduce the traffic volume from 1,200 to 1,117 vehs/hour/lane or reduce the proportion of HGVs from $20 \%$ to $17 \%$. 
Based on this contour chart, therefore, the LTA can examine the operational status of a tunnel and implement suitable risk reduction solutions.

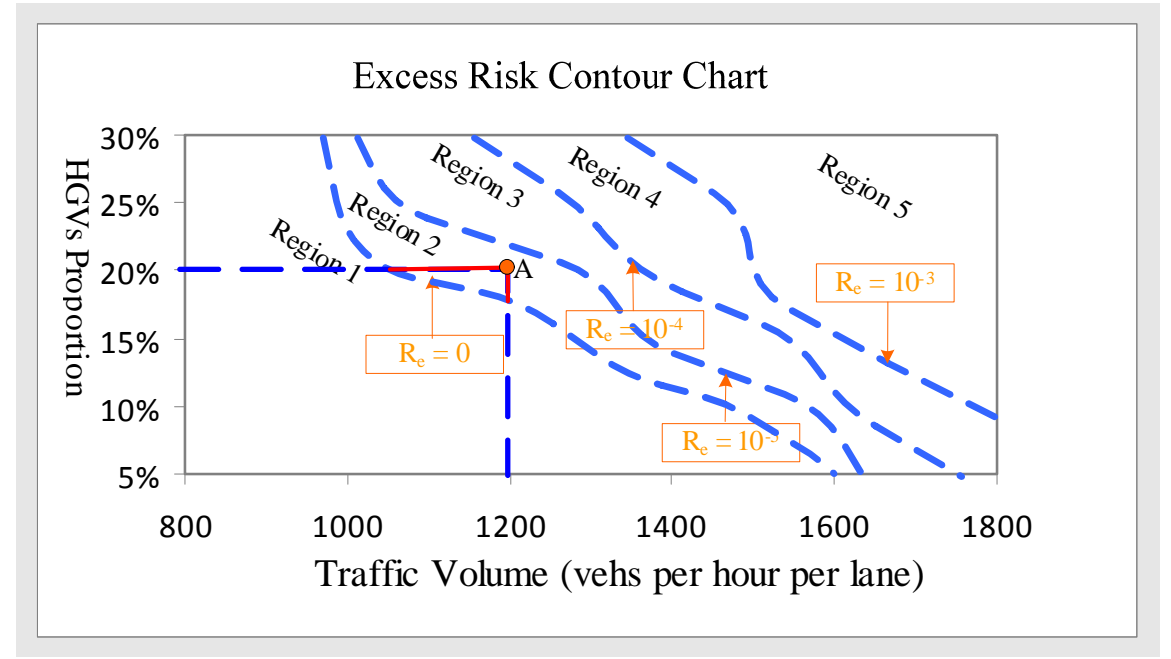

Fig. 6: Risk contour chart based on the excess risk index

\subsection{Implications for Tunnel Management}

Risk impact analysis is of great significance for tunnel management. The tunnel risk is dependent on traffic flow (traffic volume and the proportion of HGV vehicles). If a tunnel operates with an unsafe status, tunnel authorities may either reduce the traffic volume or the proportion of $\mathrm{HGV}$ vehicles passing through the tunnel. For example, three operational procedures have been considered to reduce tunnel risks in Singapore. Firstly, as presented in the introductory section, in Singapore, a form of notice is required to be submitted to the LTA for approval before an HGV can enter a road tunnel, in accordance with the Road Traffic Act. Accordingly, the LTA can insist that HGVs pass through road tunnels only during off-peak hours when traffic volumes are lower. Secondly, a tunnel entry control could be used in the entrance of the road tunnel during peak times, to ensure a safe distance of more than one hundred meters, or at least the braking distance based on the speed limit of the road tunnel. Thirdly, electronic road pricing (ERP) and ramp metering could be used to limit the traffic flow. The risk index contour chart could be used to examine the efficiency of a road pricing strategy from the viewpoint of risk reduction.

\section{CONCLUSIONS AND FUTURE WORK}


This paper has developed a QRA model-based impact analysis methodology to evaluate the impact of contributing factors on societal risk. In addition, an "excess risk index" has been defined to quantify the severities of unacceptable scenarios which place road tunnel operations above a predetermined safety target. A contour chart, based on the excess risk index, was plotted using all possible combinations of two different parameters. The contour chart can be used to help decision makers to implement suitable risk reduction solutions. Finally, the QRAFT software tool was applied to generate the F/N curves and the values of the excess risk index for the KPE road tunnel. The analysis shows that the maximum tolerable traffic volume is 1,200 vehs/hour-lane and the maximum acceptable proportion of $\mathrm{HGVs}$ is $18 \%$ of the total traffic volume.

It should be pointed out that a number of parameters are difficult to estimate precisely. Thus, there is an issue of uncertainty resulting from a lack of information and inherent variability. In the earlier study ${ }^{(4)}$, we use crisp numbers (most likely value or mean value) to represent those input parameters without considering the parameter uncertainty. Further studies should be carried out to better represent the input parameters and propagate parameter uncertainty. In reality, although the uncertainties are not taken into account in this study, the proposed methodology has more general applications to the probabilistic QRA models which take parameter uncertainty into account. Future work might carry out the risk impact analysis based on QRA models with parameter uncertainty.

\section{References}

1. PIARC Technical Committee C3.3 Road tunnel operation, 2008. Risk Analysis for Road Tunnels, May 2008.

http://publications.piarc.org/ressources/publications_files/4/2234,TM2008R02-WEB.pdf. Accessed $19^{\text {th }}$ Oct 2010.

2. Meng Q, Wang X, Qu X, Yong KT, Lee SP,Wong SC. Quantitative risk assessment models of road tunnels - state of the art and their implications for Singapore's road tunnels.

Presented at the 2nd International Tunnel Safety Forum for Road and Rail. 20-22 April 2009; Lyon: 192-203. 
3. Jonkman SN, Lentz, A, Vrijling JK, A general approach for the estimation of loss of life due to natural and technological disasters. Reliability Engineering and System Safety, 2010; 95(11): 1123-1133.

4. Meng Q, Qu X, Wang X, Yuanita V, and Wong SC. Quantitative risk assessment modeling for non-homogeneous urban road tunnels. Risk Analysis, 2011; 31(3): 382-403.

5. Land Transport Authority. Analyzing quantitative risk of road tunnels. Fact Sheet. 2010. www.lta.gov.sg/images/STARS\%20Fact\%20Sheets.pdf Accessed $19^{\text {th }}$ Oct 2010.

6. Thangasamy C, YH Wong, SP Lee, Q Meng, and X Qu. Quantitative risk analysis for Marina Coastal Expressway using software based approach. Proceedings of the 2nd World Road Congress. 26-28 Oct. 2009, Singapore.

7. Meng Q, and Qu X. Design and Implementation of a quantitative risk assessment software tool for Singapore road tunnels. Submitted to Expert Systems with Applications, 2010.

8. Aven T. On the ethical justification for the use of risk acceptance criteria. Risk Analysis, 2007; 27(2): 303-312.

9. Lemming G, Friis-Hansen P, Bjerg PL. Risk-based economic decision analysis of remediation options at a PCE-contaminated site. Journal of Environmental Management, 2010; 91: 1169-1182.

10. Horn MET, Fulton N, Westcott M. Measures of societal risk and their potential use in civil aviation. Risk Analysis, 2008; 28(6): 1711-1726.

11. Beard A, Carvel C. The handbook of tunnel fire safety. Thomas Telford Publishing. $1^{\text {st }}$ Jan 2005. London.

12. Bottelberghs PH. Risk analysis and safety policy developments in the Netherlands. Journal of Hazardous Materials, 2000; 71(1-3): 59-84.

13. Jonkman SN, van Gelder PHAJM, and J K Vrijling. An overview of quantitative risk measures for loss of life and economic damage. Journal of Hazardous Materials, 2003; 99(1): $1-30$.

14. Davis GA. Accident reduction factors and casual inference in traffic safety Studies: a review. Accident Analysis Prevention, 2000; 32(1): 95 - 109.

15. Abdel-Aty M, and A Pande. Crash data analysis: collective vs. individual crash level approach. Journal of Safety Research, 2007; 38(5): 581 - 587. 
16. Arends BJ, Jonkman SN, Vrijling JK, van Gelder PHAJM. Evaluation of tunnel safety: towards an economic safety optimum. Reliability Engineering and System Safety, 2005; 90(2-3): 217-228.

17. Dimitrakopoulos DN, Kavussanos MG, Spyrou SI. Value at risk models for volatile emerging markets equity portfolios. The Quarterly Review of Economics and Finance, 2010; 50: 515-526.

18. Rockafellar RT, Uryasev S. Conditional value at risk for general loss distributions. Journal of Banking \& Finance, 2002; 26: 1443-1471. 